\title{
Telecentro UNAH-CURLA Roatán: El primer año de una experiencia innovadora
}

\author{
Adelfa Patricia Colón \\ Centro Universitario Regional del Litoral \\ Atlántico, CURLA-UNAH
}

Martha Leticia Quintanilla

Universidad Nacional Autónoma

de Honduras- UNAH

\section{Resumen}

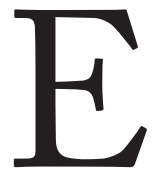

n este artículo se presenta la experiencia de la implementación de la modalidad virtual a través del Telecentro de la Universidad Nacional Autónoma de Honduras, UNAH, en Roatán, Islas de la Bahía, en su primer año de funcionamiento. Este el cuarto proyecto de este tipo que implementa la institución y sigue los principios de equidad, pertinencia y calidad.

E1Telecentro de Roatán se creó en el año 2013 siendo el Centro Universitario Regional del Litoral Atlántico, CURLA, el nodo central de la Red Educativa del Atlántico. Su objetivo es brindar una oportunidad de acceso a la educación superior a los jóvenes del departamento insular de Islas de la Bahía, donde la UNAH no tenía presencia.

Más de 50 estudiantes se forman a través del Telecentro en la Licenciatura en Ecoturismo y el Técnico en Microfinanzas en modalidad virtual. Las buenas prácticas, las lecciones aprendidas y los principales desafíos de una modalidad for- mativa innovadora para la UNAH y el país, se plantean como resultado del análisis de un proyecto de innovación educativa, que llegó a su primer año, aportando una amplia cantidad de insumos para la mejora continua de estos procesos innovadores en la educación superior hondureña.

Palabras clave: telecentro, educación virtual, equidad, innovación educativa.

Abstract: In this article the experience of the implementation of the virtual modality is presented through the telecenter of the Honduras Autonomous National University, UNAH, in Roatán, Islas de la Bahia, in its first year of functioning. This is the fourth project of this type that is implemented in the institution, and follows the principles of equality, pertinence and quality.

The telecenter of Roatán was created in the year 2013 from the Littoral Atlantic Regional University Center, CURLA, as the central node of the Atlantic educational web with the objective of bringing an opportunity of access to the higher education to the youth of the insular department of Islas de la Bahía, where the UNAH had no presence.

More than 50 students are formed through the telecenter in the bachelor's degree of Ecotourism and Microfinances in the virtual modality. The good practices, the lessons learned and the main challenges of a formative virtual modality for the UNAH and the country, are presented as a result of the analysis of a project of innovative education, that came in its first year, bringing a wide quantity of supplies for the continuous improvement of these innovative processes in the Honduran higher education.

Keywords: telecentre, online education, equity, educational innovation. $\because$ 


\section{Introducción}

La UNAH, con más de 165 años de vida institucional, es una universidad bimodal, es decir que su oferta educativa puede desarrollarse de manera presencial y a distancia, y dentro de la modalidad a distancia se incluye la educación virtual o en línea (UNAH, 2014, p.18).

En el año 2010 y en el marco de su proceso de reforma educativa y de la política de redes educativas, la UNAH presenta ante el Consejo de Educación Superior la propuesta de los Telecentros como parte de la creación de UNAH Red Virtual. Es así como mediante Acuerdo No. 2224-238-2010 se aprueba este sistema innovador que posibilita llevar educación superior de calidad eliminando barreras geográficas y espaciales, aprovechando el potencial de las tecnologías de la información y comunicación actuales.

Estos espacios buscan responder a los problemas de inequidad y acceso para la población en edad de asistir a la universidad y que habita en zonas donde la educación superior pública carece de presencia. Los datos nacionales indican que la tasa de cobertura en educación superior en Honduras es una de las más bajas de América Latina. Según la Encuesta de Hogares de mayo de 2010, asisten a la educación superior 110,026 jóvenes de 19 a 24 años y se quedan sin acceso a ésta 841,168 potenciales estudiantes. Entre estos potenciales estudiantes, hay que incluir la gran masa de jóvenes que, al no lograr concluir su educación secundaria, no tienen la posibilidad de ingresar a la educación superior (PNUD, 2012).

Para dar respuesta a necesidades regionales y mediante Acuerdo No. CT-No. 314-A-207 la UNAH aprueba la Política de Redes Educativas Regionales Universitarias, para la Gestión del Conocimiento con Calidad, Pertinencia y Equidad. (Universidad Nacional Autónoma de Honduras, 2008). De acuerdo a esta política la UNAH se organiza en ocho redes educativas: la Red de la Región Tegucigalpa, la Red de la Región Central, la Red de la Región Norte, la Red de la Región Oriental, Red de la Región Sur Oriental, Red de la Región del Litoral Atlántico, Red de la Región Occidental y la Red de la Región Sur (Universidad Nacional Autónoma de Honduras, 2008).

La Red de la Región del Litoral Atlántico abarca los departamentos de Atlántida, Colón, Islas de la Bahía y Oriente de Yoro; concentra la región del Litoral Atlántico, la región del Río Aguán y parte de la región del Río Plátano-Tinto o $\mathrm{Ne}$ gro. Atender las demandas de educación superior de la zona insular del país es una responsabilidad de esta Red, integrada por el Centro Universitario Regional del Litoral Atlántico (CURLA) con sede en La Ceiba, el Centro Universitario Regional del Valle del Aguan (CURVA) con sede en Olanchito, Yoro, el CRAED de Tocoa, el Centro Experimental de Sinaloa, Colón y el Instituto Tecnológico de Tela.

\section{Creación del Telecentro UNAH CURLA-Roatán}

El Telecentro UNAH-CURLA Roatán surge como iniciativa presentada por la UNAH a la municipalidad y fuerzas vivas de la Isla de Roatán que demandaban la presencia de esta casa de estudio en esa región.

Esta necesidad impostergable de la presencia de la universidad estatal más grande del país en una zona de gran desarrollo turístico nacional fue analizada en una reunión de trabajo sostenida por las autoridades edilicias, las autoridades de la UNAH y representantes de Islas de la Bahía en el Congreso Nacional en el mes de abril del año 2013.

A esa fecha la UNAH ya registraba experiencia en ampliación de cobertura educativa universitaria a través de un proyecto de innovación educativa que bajo los principios de equidad, calidad y pertinencia tenía en funcionamiento tres telecentros: UNAH VS Choloma y UNAH VS en Puerto Cortés, ambos situados en la Red del Valle de Sula, y el Telecentro CUROC-Gracias, Lempira en la Red Educativa de Occidente.

Con base en esta experiencia la UNAH propuso a la Municipalidad y sectores de la sociedad civil de Roatán la apertura de un Telecentro, figura académica innovadora que llegaría a la zona con una modalidad educativa propia del siglo XXI, para contribuir a reducir la brecha de inequidad educativa y digital y ampliar la cobertura de la UNAH hacia ese departamento insular.

La oferta de carreras propuesta fue el Técnico en Microfinanzas que ya se ofertaba en modalidad virtual en otros telecentros y forma profesionales para desarrollar el sector de la pequeña y mediana empresa, uno de los rubros que sostiene la economía del país. Y la Licenciatura en Ecoturismo, carrera que la UNAH oferta sólo en el CURLA, para la formación de recurso humano en áreas profesionales coherentes con el desarrollo humano, científico y tecnológico que demandan las nuevas tendencias sociales y económicas de la zona, considerado como polo del desarrollo turístico.

El modelo de telecentros y la oferta virtual se presentó a las fuerzas vivas de Roatán a través de un taller realizado en el 16 de mayo de 2013 donde participaron representante de la municipalidad y de todos los sectores de la sociedad civil de esa isla. 
Por parte de la UNAH participó la Dirección y el equipo académico del CURLA; la Vicerrectoría Académica, la Dirección del Sistema de Admisiones y la Dirección de Innovación Educativa. Resultado de ese taller se estableció y consensuó la ruta crítica para la puesta en funcionamiento del Telecentro UNAH CURLA Roatán.

Siguiendo el modelo de telecentros de la UNAH, que establece poner en funcionamiento estos establecimientos en alianza estratégica con las municipalidades, la Alcaldía de Roatán designó vía resolución de corporación municipal asignar la planta superior del edificio donde funciona la biblioteca pública Jared Hynds, ubicada en Frech Harbor, asimismo una transferencia de tres millones de lempiras para la adquisición del equipo de cómputo, educacional, mobiliario y desarrollos educativos innovadores, como inversión de la primera fase del proyecto.

Este lugar fue inspeccionado por el equipo de ingenieros de la Secretaria Ejecutiva de Infraestructura y Proyectos de Inversión, SEAPI, instancia universitaria que elaboró y ejecutó el plan de remodelación siguiendo las exigencias pedagógicas, de seguridad, físicas y tecnológicas exigidas por la máxima casa de estudios del país para los espacios formativos de esta índole. La parte de red de datos e instalación del equipo de cómputo estuvo a cargo de la Dirección Ejecutiva de Gestión de Tecnologías, DEGT y el equipo de ingenieros en sistemas de la Dirección de Innovación Educativa. La alcaldía de Roatán asumió financieramente la remodelación de las instalaciones del Telecentro.

\section{La puesta en marcha}

El mes de mayo de 2013, marcó el inicio de la presencia de la UNAH en Roatán. La Universidad y el grupo gestor del Telecentro UNAH CURLA-Roatán (Municipalidad y fuerzas vivas) se propusieron una ruta crítica que de acuerdo al calendario académico propuso como meta el funcionamiento del telecentro en el mes de noviembre de ese mismo año para que los primeros alumnos se matricularán en el I Periódo Académico 2014.

Ello implicó un trabajo intensivo y el desarrollo de tareas simultáneas orientadas a la firma de carta de entendimiento UNAH-Alcaldía, readecuación de espacio físico, desarrollos académicos, adquisición de equipo tecnológico y educacional, el censo de aspirantes a estudiar en la UNAH, la socialización de las fechas para realizar la Prueba de Aptitud Académica, PAA, cuya aprobación es requisito de admisión, la capacitación y selección de docentes para la virtualización de asignaturas de los diversos departamentos implicados en la nueva oferta de carreras virtuales del CURLA.

\section{Resultados}

\section{La PAA en Roatán}

Uno de los resultados de este proceso fue el logro que la Prueba de Aptitud Académica, PAA, se realizara

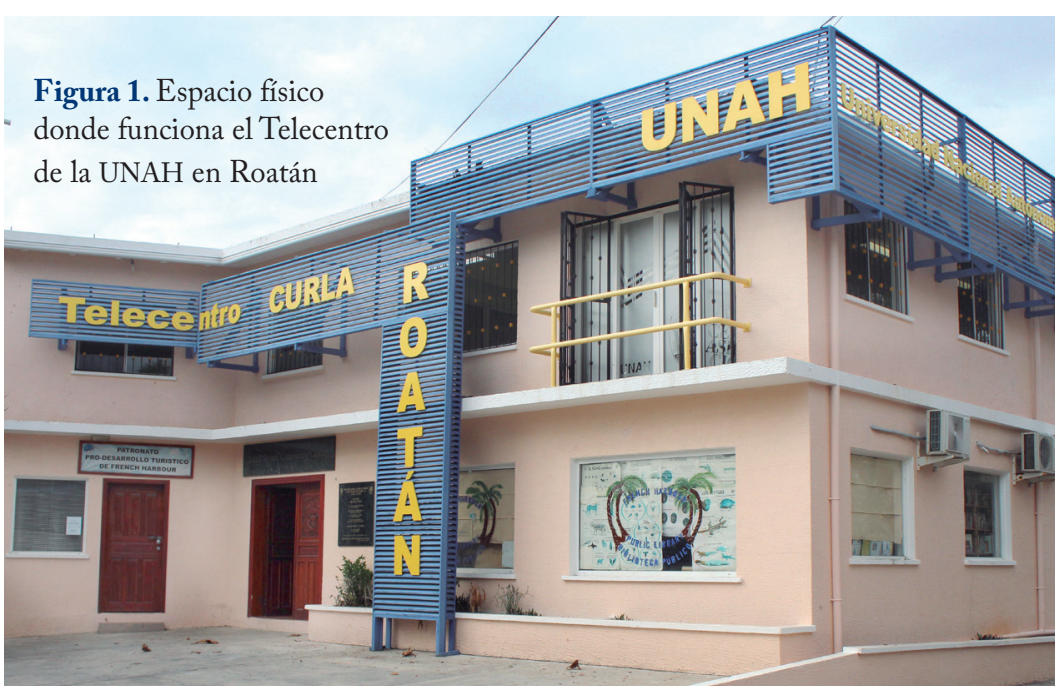

Fuente: Dirección de Innovación Educativa-UNAH en Roatán, dado el costo que significaría a los aspirantes trasladarse a practicarla hasta el CURLA en la ciudad de La Ceiba. Ello implicó que la Dirección del Sistema de Admisiones, DSA, inspeccionará los posibles centros educativos para determinar si reunían las condiciones de seguridad que se exigen para este tipo de pruebas. Asimismo, el compromiso de la Alcaldía Municipal de apoyar la estadía del equipo académico responsable de esa tarea.

Es así como la primera PAA se realizó en Roatán el 13 de julio de 2013 en la Escuela Modelo de Sandy Bay, siendo el primer lugar externo a los Centros Regionales autorizado por la DSA de la UNAH para practicar la PAA.

La capacidad de gestión académico-administrativa y de desarrollo de la UNAH se puso a prueba y en seis meses, mayo a noviembre de 2013, el Telecentro UNAH CURLA-Roatán estaba funcionando, específicamente el 21 de noviembre, fecha que se inauguró el inicio del curso propedéutico para el aprendizaje en línea "No creí que la UNAH iba a cumplir" expresó el alcalde de Roatán Julio Galindo cuando acompañó a los aspirantes a ingresar a la UNAH y a padres de

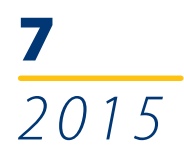


Tabla 1. Docentes del CURLA capacitados para la docencia en línea CURLA 2013-2014.

\begin{tabular}{|c|c|}
\hline Departamento académico & No. docentes capacitados \\
\hline Química & 3 \\
\hline Biología & 3 \\
\hline Matemática & 3 \\
\hline Ciencias Sociales & 6 \\
\hline Humanidades & 6 \\
\hline Ecoturismo & 12 \\
\hline Administración de Empresas & 1 \\
\hline Economía Agrícola & 8 \\
\hline
\end{tabular}

Fuente: elaboración propia a partir de registro estadístico DIE-UNAH

Tabla 2. Docentes del CURLA capacitados para el diseño y desarrollo de asignaturas virtuales 2013-2014

\begin{tabular}{|c|c|c|}
\hline & Departamento Académicos & No. docentes capacitados \\
\hline & Ciencias Sociales & 4 \\
\hline & Humanidades y Artes & 6 \\
\hline & Administración de Empresas & 5 \\
\hline & Economía Agrícola & 2 \\
\hline & Biología & 7 \\
\hline & Química & 3 \\
\hline 2015 & Matemática & 2 \\
\hline
\end{tabular}

Tabla 3. Estadísticas del proceso de admisión y matrícula en el Telecentro UNAH-CURLA Roatán

\begin{tabular}{|l|c|c|c|}
\hline \multirow{2}{*}{ Proceso } & \multicolumn{3}{|c|}{$\mathbf{2 0 1 4}$} \\
\cline { 2 - 4 } & I & II & III \\
\hline Inscritos en la PAA & 87 & 31 & 40 \\
\hline Presentaron documentos & 57 & 6 & 26 \\
\hline Realizaron la PAA & 45 & 6 & 18 \\
\hline Aprobaron la PAA & 23 & 4 & 6 \\
\hline $\begin{array}{l}\text { Inscritos en Curso propedéutico } \\
\text { para el aprendizaje en línea }\end{array}$ & 62 & 31 & 6 \\
\hline Acreditaron c. propedéutico & 51 & 17 & 6 \\
\hline Primer ingreso & 53 & 12 & 9 \\
\hline Reingreso & 0 & 24 & 30 \\
\hline Licenciatura en Ecoturismo & 13 & 5 & 6 \\
\hline $\begin{array}{l}\text { Técnico Universitario en Micro } \\
\text { finanzas }\end{array}$ & 40 & 31 & 33 \\
\hline Total Matriculados & $\mathbf{5 3}$ & 36 & 39 \\
\hline
\end{tabular}

Fuente: Elaboración propia a partir de datos de la DIPP y la DSAde la UNAH familia al primer día de funcionamiento del telecentro.

Los estudiantes que aspiran estudiar en la modalidad virtual deben cubrir un paso más en relación con los de la modalidad presencial, ya que previamente deben aprobar un curso propedéutico que los capacita tecnológica y pedagógicamente para la educación en línea, herramienta de apoyo para el estudio en una metodología donde el proceso formativo es mediado por las TIC y por el docente tutor o asesor.

Este curso comenzó en el Telecentro de Roatán con 58 alumnos que habían aprobado la PAA realizada el 13 de julio. De estos estudiantes, 11 aplicaron para estudiar la licenciatura en Ecoturismo y 47 el Técnico en Microfinanzas de acuerdo al reporte de la Dirección del Sistema de Admisiones (DAS, 2013).

Para el CURLA, creado en 1967 y por ende para los Departamentos de Ecoturismo y el área ecónomo administrativa, así como para los Departamentos que ofrecen clases a estas carreras, asumir una nueva modalidad educativa significó un desafío superlativo: debían generar nuevos ambientes de aprendizaje mediados por las TIC y además prepararse para el ejercicio de la docencia en línea, todo partía por implicarse en procesos de capacitación que para ello la DIE ponía a su disposición.

Como resultados de las capacitaciones respectivas se capacitaron entre 2013 y 2014, 42 profesores para la Asesoría en Línea como se muestra en la Tabla 1 para el diseño y desarrollo de asignaturas en línea 32, como se observa en la Tabla 2.

\section{Estudiantes}

En el primer año de funcionamiento, el 2014 se matricularon 77 
estudiantes, de los cuales 18 pertenecen a la carrera de Ecoturismo y 59 a la carrera de Técnico en microfinanzas. Sin embargo, para el III periodo académico la carrera de Ecoturismo sólo contaba con 6 estudiantes: 3 del grupo A, 1 del B y 2 del C; mientras que el técnico en Microfinanzas contaba con 33 estudiantes. Esto totalizaba 39 estudiantes activos durante el III periodo académico del 2014.

En la Tabla 3 se muestra la dinámica de los estudiantes durante el 2014, desde el momento que se inscriben para realizar la PAA hasta su proceso de matrícula durante el tercer periodo 2014.

La Tabla 3 refleja que hay una diferencia significativa entre los que se inscriben para realizar la PAA y los que culminan el proceso de pago y presentación de documentos. Sin embargo, estas cifras son similares en toda la UNAH independientemente de la modalidad o centro de estudio.

Otro elemento de análisis que se deriva de los datos mostrados es que la transición de un modelo formativo presencial, altamente innovador y mediado por las TIC, será un proceso lento, que implicará la aplicación de estrategias también innovadoras para que los estudiantes se apropien de las competencias y habilidades que exige la modalidad virtual. Asimismo, actividades que muestren las ventajas y beneficios de esta nueva modalidad.

Para determinar qué factores socioculturales pueden favorecer el crecimiento de la matrícula en el Telecentro UNAH CURLA Roatán, o cuáles están afectando el aprovechamiento de esta posibilidad de formación universitaria, será necesario llevar a cabo investigaciones que posibiliten una estrategia de intervención científica y efectiva.
Empíricamente se puede observar en los estudiantes que ya son parte del Telecentro, el deseo de valorar esta oportunidad como algo único, sin embargo la modalidad virtual exige mayor compromiso y tiempo, tanto de los estudiantes, como de los docentes. Aún existen obstáculos que subsanar para que realmente las ventajas que ofrece este sistema puedan ser aprovechadas por ambos actores del proceso formativo y la modalidad se convierta en una alternativa real para un mayor número de estudiantes.

Transformar el modelo de enseñanza clásico, completamente presencial y asumir un nuevo modelo de aprendizaje donde la formación virtual pueda completar e incluso sustituir a la formación tradicional es todo un reto, como lo señala Nieto Goller (2012):

Bajo el aspecto pedagógico y cognoscitivo que encierra la educación "virtual", abierta y a distancia, y la irrupción de las aclamadas "competencias", el "aprendizaje significativo", etc., de la mano del proceso de la globalización, la dialéctica emprendida por aquellos a favor y los que se encuentran en contra de ella, gira, en primer lugar, la cuestión de si el navegar es o no aprender. De idéntica forma, el que si la interactividad en Internet genera o no aprendizajes. Le sigue el dilema de si buscar información en Internet es fácil o no tanto. Seguidamente nos topamos con aquello de que si la escuela o institución con Internet es moderna o sigue siendo la misma. Acto seguido hace su aparición el sí comunicarse en línea (online) resulta en una mejor comunicación humana. Luego nos vamos a cosas mayores, como si la hipermedialidad de Internet, es decir, su no-linealidad favorece los procesos de aprendizaje (pág. 145).
Todos estos aspectos se han visto reflejados en los diferentes actores $\mathrm{du}-$ rante el primer año en funcionamiento. Se han presenciado reacciones variadas: mucha esperanza y alegría en la población de Roatán, optimismo en las autoridades centrales de la universidad, escepticismo por parte de algunos docentes del CURLA, y preocupación en las autoridades encargadas de implementar la modalidad, ante el reto docente y estudiantil de asumir el paradigma formativo del presente siglo.

Al respecto Cabero (2006) citado por Padilla-Beltrán, Vega-Rojas y Ricón-Caballero (2014), ya advierte algunas dificultades que se presentan en la implementación de procesos formativos mediados por las TIC a nivel universitario, por ello considera que profesores y estudiantes deben modificar ciertos hábitos de interacción y acercamiento al conocimiento, ya que estos nuevos ambientes de aprendizaje exigen una serie de habilidades tecnológicas y pedagógicas que habrán de considerarse obligadamente.

El estudiante puede formarse para una transición del paradigma de enseñanza tradicional a uno más centrado en la autonomía y el metaprendizaje. La calidad de la formación puede no ser la esperada, pues si no se tienen las consideraciones necesarias para la transición de una educación tradicional a una sustentada en el discurso de TIC, se puede incidir en la pérdida progresiva del discurso pedagógico. Las TIC como oportunidad de seguimiento constante del aprendizaje, demandan mayor cantidad de tiempo en aspectos de tutoría a los estudiantes, por ende, el docente emplea más esfuerzo para la orientación y la motivación del grupo de aprendizaje (pág. 284). 
Otro elemento que se evidencia en esta experiencia de innovación educativa del CURLA, es que el paradigma del espacio de formación presencial, como único para el aprendizaje, está estacionado fuertemente aún en la universidad y el desaprendizaje de este modelo tradicional del siglo $\mathrm{XX}$, registra una dinámica muy lenta para la adopción del modelo formativo del siglo XXI, lo que se evidencia para el caso, en el escepticismo y poco valor que algunos gestores académicos de la UNAH dan a los espacios formativos mediados por las TIC y al trabajo de la docencia en línea. De ahí que es necesario que a todo nivel en la UNAH haya reconocimiento del esfuerzo, dedicación y compromiso que requiere una tutoría o asesoría en línea.

\section{Conclusiones}

Al dar una mirada precisa sobre el proyecto de innovación educativa

de la UNAH en Roatán a través del CURLA, como nodo central de la Red Educativa del Litoral Atlántico, se pueden extraer algunas conclusiones generales:

La apertura y puesta en funcionamiento del Telecentro UNAH CURLA-Roatán se constituye un acontecimiento histórico para la población de esa Isla caribeña hondureña y para la Universidad, ya que la presencia de la máxima casa de estudios del país era un sueño para los pobladores de Roatán. En sus 166 años de fundación la UNAH decide surcar el territorio nacional de Roatán.

Se resalta la convicción de las autoridades de la Municipalidad de Roatán en la educación como factor de desarrollo de esa Isla, al suscribir una carta de entendimiento con la UNAH y asumir las responsabilidades que el modelo de los Telecentros de la UNAH establece para la puesta en marcha de esos espacios de aprendizaje.

La brecha entre los que se inscriben para realizar la PAA, los que logran matricularse, es muy grande, por lo cual se deben realizar investigaciones que permitan tomar acciones que contribuyan a disminuir la diferencia y que más jóvenes de la Isla de Roatán aprovechen la presencia de la UNAH en ese sector.

Aunque muchos docentes ya han recibido capacitación en el manejo de aulas virtuales y el diseño de asignaturas en línea, es necesario fortalecer tanto la estrategia y desarrollos educativos de los espacios de aprendizaje o asignaturas en línea y la presencia del docente en el espacio de aprendizaje en línea. Una buena docencia en línea supera cualquier debilidad que se registre a nivel de los espacios de aprendizaje mediados por las TIC, de ahí que mientras no se apliquen mejoras a los actuales espacios formativos en línea la presencia del tutor es vital para el buen desempeño del estudiante. Ser tutor en línea requiere un alto nivel de compromiso y el reconocimiento por parte de las Jefaturas de Departamento y Coordinaciones de Carrera.

\section{Referencias}

Dirección de Innovación Educativa-UNAH. (2014). Telecentros UNAH. Recuperado de: https:// die.unah.edu.hn/servicios-educativos/telecentros-unah/

Nieto Göller, R. A. (2012). Educación virtual o virtualidad de la educación. Revista Historia de la Educación Latinoamericana.

Padilla-Beltrán, J., Vega-Rojas, P., y Rincón-Caballero, D. (2014). Tendencias y dificultades para el uso de las TIC en educación superior1. Entramado, 10(1). Recuperado de: http://www.scielo.org.co/scielo. php?script=sci_arttext\&pid=S0038032014000100017\&lang=pt

PNUD. (2012). Informe sobre desarrollo humano en Honduras 2011. Reducir la inequidad, un desafío impostergable. Tegucigalpa.

Universidad Nacional Autónoma de Honduras. (2008). Redes Educativas Regionales de la UNAH para la gestión del conocimiento con calidad, pertinencia y equidad (serie de publicaciones de la reforma universitaria No.2). Tegucigalpa, Honduras.

Universidad Nacional Autónoma de Honduras. (2011). El modelo de educación virtual y de Telecentro Universitario de la UNAH. Tegucigalpa, Honduras.

Universidad Nacional Autónoma de Honduras. (2014). Serie de Publicaciones de la Reforma Universitaria No.5. Recuperado de: La Educación a Distancia en la UNAH: file://C:/Users/HP/ Downloads/No.-5-Documento-Conceptual-EaD.pdf

Vicerrectoría Académica de la UNAH. (2009). El Modelo Educativo de la UNAH. Tegucigalpa: Ciudad Universitaria "José Trinidad Reyes".

Recibido: 07/08/2015 Aceptado: 28/09/2015 